\title{
Notes toward a verifiable vector algebraic basis for colorimetric modeling
}

\section{Document Version}

Accepted author manuscript

Link to publication record in Manchester Research Explorer

\section{Citation for published version (APA):}

Oulton, D. P. (2009). Notes toward a verifiable vector algebraic basis for colorimetric modeling. Color Research and Application, 34(2), 163-169.

\section{Published in:}

Color Research and Application

\section{Citing this paper}

Please note that where the full-text provided on Manchester Research Explorer is the Author Accepted Manuscript or Proof version this may differ from the final Published version. If citing, it is advised that you check and use the publisher's definitive version.

\section{General rights}

Copyright and moral rights for the publications made accessible in the Research Explorer are retained by the authors and/or other copyright owners and it is a condition of accessing publications that users recognise and abide by the legal requirements associated with these rights.

\section{Takedown policy}

If you believe that this document breaches copyright please refer to the University of Manchester's Takedown Procedures [http://man.ac.uk/04Y6Bo] or contact uml.scholarlycommunications@manchester.ac.uk providing relevant details, so we can investigate your claim.

\section{OPEN ACCESS}




\title{
COLOR FORUM
}

\section{Notes Toward a Verifiable Vector Algebraic Basis for Colorimetric Modeling}

\author{
David P. Oulton*
}

School of Materials Science, Manchester University, United Kingdom

Received 31 August 2007; revised 13 December 2007; accepted 19 February 2008

\begin{abstract}
The presented notes aim toward improved models of the scalar visual response to flat-field stimuli, and are prompted by unease over the complexity of existing colour difference models. Some of the basic assumptions of colorimetry are examined in detail, and analytical methods whereby these assumptions can be investigated experimentally are presented. A key finding is that the standard CIE colorimetric model is verifiably correct as a predictor of point colour identity and metameric visual equivalence. It is however demonstrated to be a misleading basis for modeling colour difference, and an alternative scalar reference basis and experimental analysis method are presented (c) 2009 Wiley Periodicals, Inc. Col Res Appl, 34, 163-169, 2009; Published online in Wiley InterScience (www.interscience.wiley.com). DOI 10.1002/col.20479
\end{abstract}

Key words: colour difference; colormetric modeling; affine geometry; scalar and ratio value visual responses

\section{INTRODUCTION}

The motivation for offering these notes is a deep unease with the complexity of existing flat-field colour difference models and their reliance on essentially empirical data fitting. I also deplore the reliance of many investigators on the co-ordinates of CIE XYZ colour space as a scalar reference basis for analyzing their results and building their models. I therefore invite readers of the Journal to debate the need for an alternative basis for colour difference modeling. The debate I believe will be between two dis-

\footnotetext{
*Correspondence to: David P. Oulton (e-mail: david.oulton@manchester. ac.uk).

() 2009 Wiley Periodicals, Inc.
}

tinct philosophical positions. On one hand, we have those who believe that the human visual response has no specific mechanism that quantifies colour difference, and in consequence the sensation is the result of trainable guesswork. The proponents therefore regard any attempt to find a representative model of this response as either pointless or doomed to failure. This stance is well documented in Donald Laming's book "Measurement of Sensation."1 There are on the other hand those of us who expect that tangible explanatory benefit or better quantitative description can be delivered by improved colorimetric models. ${ }^{2,3}$

In presenting these notes I seek firstly to focus this debate, and secondly to introduce a new approach to experimental design, and to colorimetric analysis that in principle could resolve the debate authoritatively. The new analysis is essentially neutral in that it is equally applicable to generating support for either position, but it will become clear that intuitively I expect the debate to be resolved in favor of tangible benefit: I also believe however that a substantial investment in new experiments will be necessary to demonstrate that this intuition is sound. In the following discourse I suggest in outline how the relevant experiments could be designed and executed, and the hypothesis is that the debate can be resolved incrementally by building up a set of definitive linearized models for the component visual responses.

\section{COLORIMETRIC ANALYSIS BY UNIT VALUE REDEFINITION AND PROJECTIVE MAPPING}

All possible visual stimuli are characterized by reference to the radiometric scales of the physical domain. In this domain the additive value and quantitative equivalence properties are fundamentally constant, and this is the basis for the real number scale and the axiomatic definitions of 


\section{A NOTIONAL HIERARCHY of PHOTOPIC VISUAL RESPONSES}

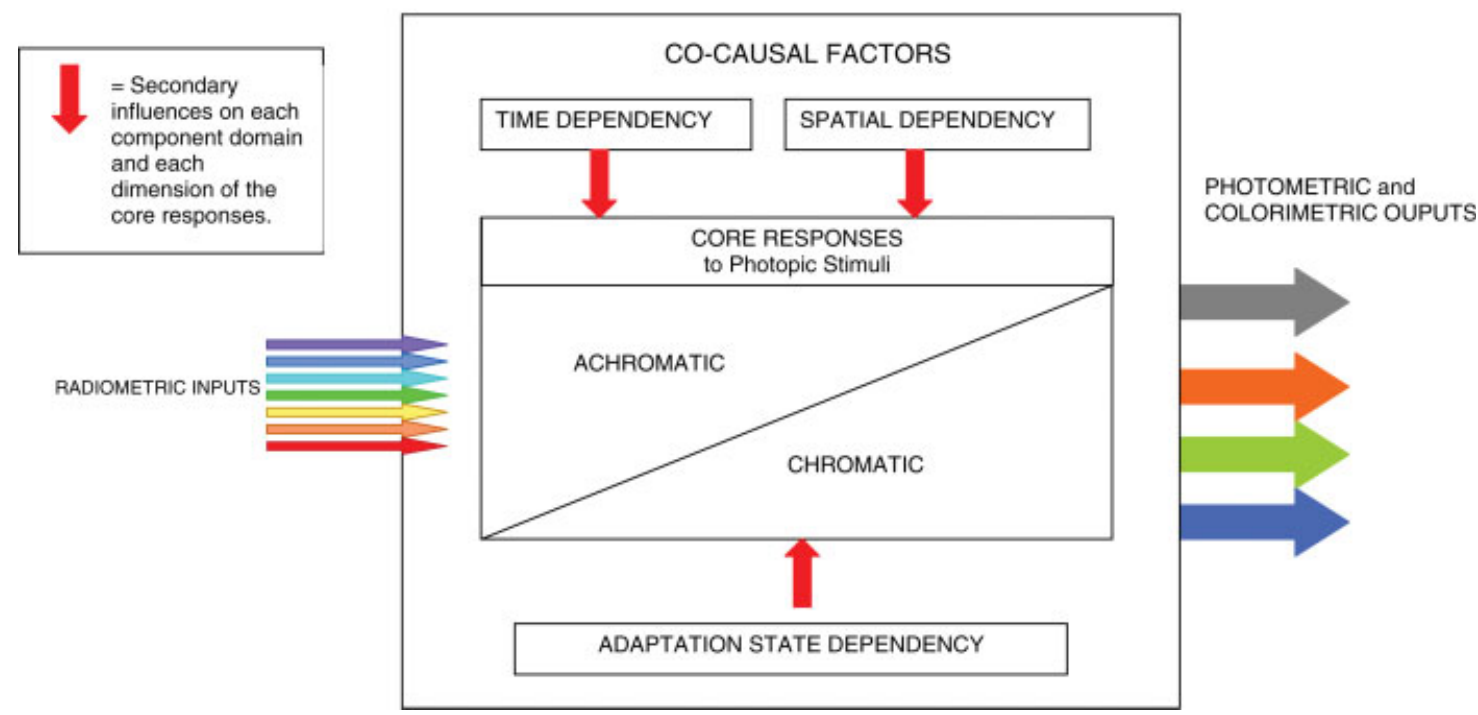

FIG. 1. The notional hierarchy of components and relationships of the photopic visual response that is used to illustrate the analysis. [Color figure can be viewed in the online issue, which is available at www.interscience.wiley.com.]

vector analysis: It is also the basis for the use of internationally accepted unit value definitions, and for the equations and laws of Newtonian physics that quantify the relationships between physical cause and effect. In fields such as colorimetry, however, there are many scalar visual responses including that of colour difference that are only measurable by inference from their unit cause. The visual responses to radiant stimuli are therefore conventionally expressed in tristimulus units rather than units of photopic response such as units of hue or lightness difference: We then interpret the resulting colour spaces to indicate the presence of intrinsically complex scales of visual difference. In these notes I will hypothesize alternatively that this apparent complexity actually reflects the combined effect of three or more essentially simpler component relationships, and that these relationships arise from and indicate differences in unit value in the distinct sub-component domains of the visual response.

Under this hypothesis I next adopt the concept of "finding the linear model" suggested by Berns ${ }^{4}$ as a key analytical method, and within this method the strategy is to identify and quantify distinct sets of domain specific constants. Some of these constants must quantify unit scalar value and may have a nonlinear definition relative to unit cause as in the CIE $L^{*} a^{*} b^{*}$ model, and others must characterize stimulus ratios and metameric visual equivalence sets as in the CIE XYZ Standard Observer model. The presented colorimetric hypothesis thus closely parallels the standard approach; however I seek a more detailed and verifiable model using a notional hierarchy of domain-specific sub models as illustrated in Figs. 1 and 2. The relevant scalar and proportionate effect relationships are then evaluated using targeted experimental data sets, and a nonlinear variant of the "projection onto convex sets" or POCS analysis method described by
Stark and Yang $^{5}$ is used, in order to isolate and quantify the component relationships.

Figures 1 and 2 are intended to highlight potentially important inter-domain boundaries where innate differences in unit value may exist and unit value transformation may be necessary. They should not be interpreted as a model of the human visual response. In principle such a model may eventually emerge, but only if future experiment does indeed validate additional visual response constants and these can also be related successfully to specific response mechanisms.

\section{Vector Addition and Numeric Scaling}

A clear theoretical and experimental distinction is next established between the processes and axiomatic definitions of vector addition and those of numeric scaling. The axioms of affine geometry establish two subsets of properties. The first subset is quantified by the multidimensional constants that characterize order, ratio value, vector direction, and point-identity equivalence. These are the constants of spatially oriented proportionate effect and vector addition, and under the defining axioms they are independently valid, provided only that they model a domain within which scalar value is strictly constant. The second subset is of scalar properties, and its members must be quantified either by declaring or experimentally characterizing unit value within each dimension of the vector system. The axiomatic distinction is between the vector system that describes the multidimensional additive properties of a given domain, and the definitions of unit value that scale it. It follows that the unit definition must be a constant of all experiments that are used to determine vector additive ratio value, and that the ratios and directional dependencies of vector addition must be treated as constants in any experiments that quantify the numeric scaling of the vector system. 


\section{of the CORE RESPONSE}

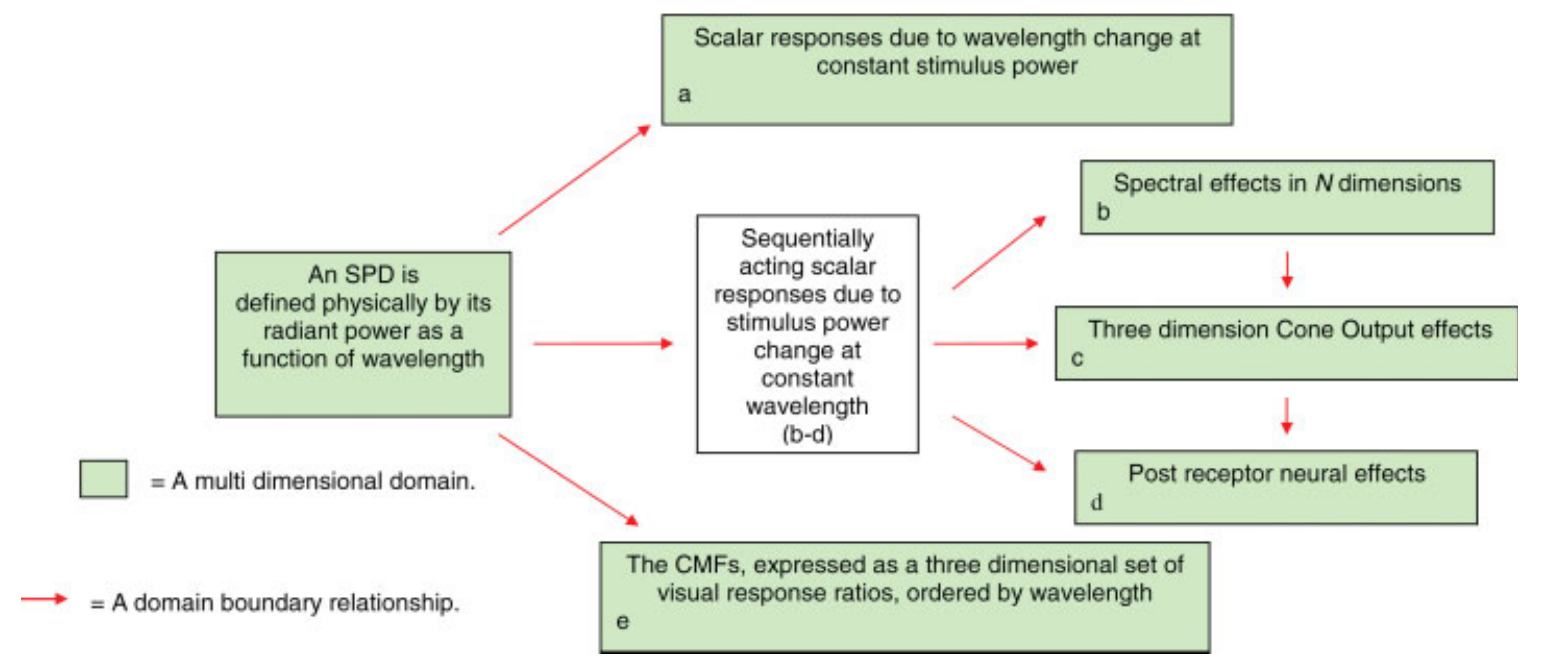

FIG. 2. Some illustrative sub-divisions of the core steady-state perceptual response to a given stimulus power density and wavelength at constant adaptation and in a constant spatial context. [Color figure can be viewed in the online issue, which is available at www.interscience.wiley.com.]

Thus far we have obtained two significant analytical results. First, we have classified and isolated the subset of multidimensional vector defining properties, and verified that they are independently quantifiable by constants such as the Colour Matching Functions or CMFs. We have thus established a valid theoretical basis for predicting point colour identity accurately by vector sum as in the CIE system. Second, we have isolated the scalar response as a distinct and independently quantifiable property that must be modeled by a set of one or more single (or intra) dimension scales. It follows that the CIE Standard Observer model and its defining datasets provide an axiomatically verifiable characterization for ratios of proportionate effect, instances of visual colour order, and instances of metameric visual equivalence. It also follows that the point identities thus defined can be redistributed in colour space by altering the individual unit value scales. In effect the CIE $L^{*} a^{*} b^{*}$ model already does this, but an improved multi-stage solution is sought via the suggested analytical and experimental method. The intent is to ensure that the visual scaling in each dimension of the response is independently quantified by correctly designed experiments; to increase the dimensionality of unit definition (as highlighted in Fig. 3); and to use sequential unit redefinition when it is necessary to distinguish between the properties of consecutively acting transformations within the overall response (as suggested in Fig. 2).

\section{Altering the Quantifying Scalar Basis of the Colorimetric Analysis}

The presented method uses nonlinear projection onto convex sets to extend the vector analysis of additive col- our mixing published by Krantz. ${ }^{6,7}$ The axioms of Krantz's analysis (see also Appendix) define what are known as Grassmann structures, and they specify affine vector systems where Grassmann's laws of colour mixing hold true. Apparent failures of Grassmann additivity in the visual response are however well documented by Alman, Pridmore, Thornton, Kuehni ${ }^{8-11}$ and others. The axioms of affine geometry quoted by Krantz are therefore used to specify a set of reference Grassmann structures, and such failures of additivity are then quantified and calibrated as numeric deviations from Grassmann's Laws by projecting the relevant dataset values onto this reference space definition.

The axiomatically uniform numeric scaling within each reference dimension is declared to represent uniform increments of the visual response, and I will therefore refer to each reference space as a Uniform Colour System (or UCS structure), in order to emphasize its status as a definition of both visual and numeric uniformity. The adopted sequence of initially $N$-dimensional and later three-dimensional reference spaces is denoted in Fig. 3, which also highlights the increased number of scaling dimensions that is used in the analysis.

I emphasize at this point that the component UCS structures are used only as reference spaces or templates for constructing a colour space. They must not be confused with the resulting experimentally quantified colour space models. These are described later in Section "Practical realization of the analytical method" when the practical methods for mapping experimental datasets onto UCS reference structures are elaborated.

Under the presented analysis, the CIE XYZ co-ordinate system is validly defined three dimensionally by reference to a triplet of mono-chromatic primaries, and the 


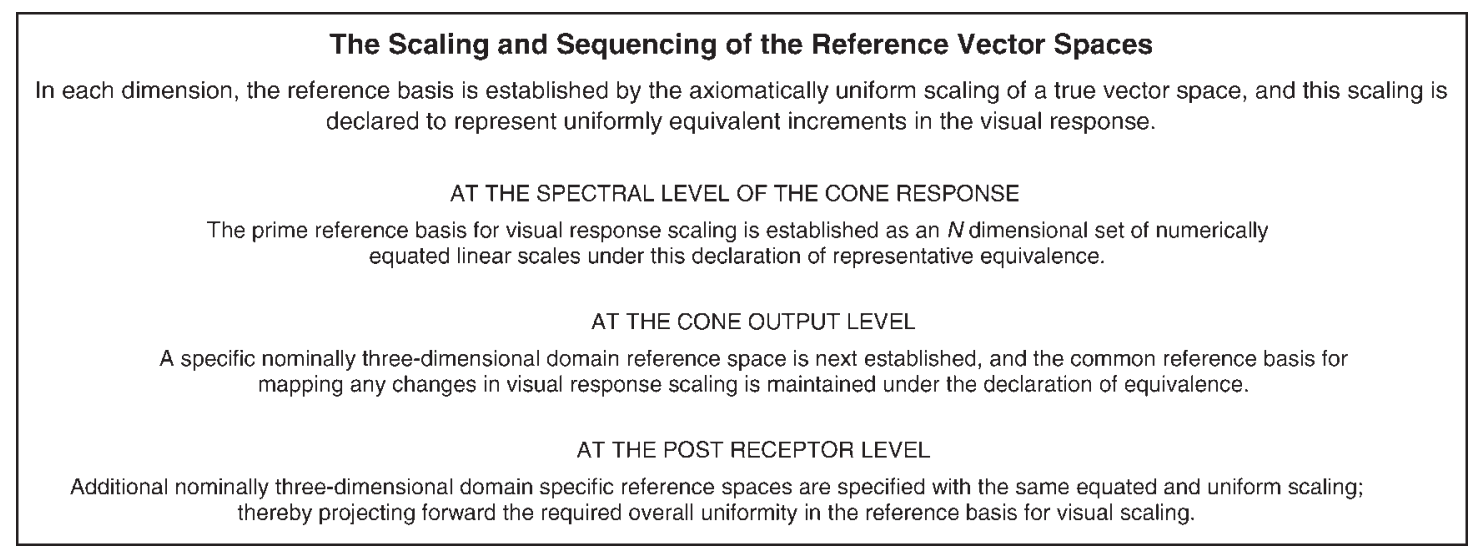

FIG. 3. The establishment of a common reference basis for visual response scaling over all sub-domains is illustrated. Some of the domain specific members of the set of reference vector spaces are identified, and the required dimensionality of reference scaling at each level is also indicated. The dimensionality at the post spectral levels is however an indicative minimum and it may need to be increased to reflect the number of independent parameters that are found experimentally to alter the observed response significantly.

constancy of scaling over affine transformation is provided by the physical stimulus values. Scalar value is then equated inter-dimensionally by normalization at the neutral balance axis and by reference to the uniform spectral power distribution (or SPD) of Illuminant SE. The visual response model is then validly weighted (again three dimensionally) by the spectrally defined CMF ratios. It should be noted however that the above definition is based exclusively on visual matching experiments, and the visual difference scale is then an undefined constant that cancels out across of the quantifying visual match equations. It follows that the defining datasets only quantify the properties of visual equivalence and ratio value, and quite correctly the scaling continues to be expressed in terms of physical units that are re-weighted by experimentally observed proportionality ratios.

When the colour difference results from MacAdam's colour matching error (or CME) experiment ${ }^{12}$ are thus scaled by unit tristimulus value as in the CIE $x, y$ plane, it is necessary to resort to Riemannian geometry to model them, or to use locally valid ellipsoid parameters as in CIE $L^{*} a^{*} b^{*}$ space. I hypothesize however that this is firstly because the unit value of many visually distinct dimensions and sequentially acting responses are modeled by a single undifferentiated co-ordinate system, and secondly because the co-ordinates are established by serial transposition of the quantifying unit value axes. However, under the presented analysis the proportionality ratios of the Standard Observer CMFs can be regarded as an independently valid set of constants, we may therefore choose an alternative visually more meaningful scalar reference basis for calibrating the unit visual difference scales.

The chosen alternative is provided by our set of axiomatically defined reference spaces, and we then effectively invert the conventional procedure for constructing a colour space. Thus, rather than extrapolating forward from sets of CIE XYZ co-ordinate tristimulus values, we hypothesize that the UCS reference space is a true model of the properties under investigation. Scalar matrix mapping onto this space is then used to establish whether or not the uniform and constant properties thus represented can also be validated as statistically significant properties of the relevant data sets.

\section{THE SCALAR VALUE ANALYSIS}

Under the theory of projection onto convex sets, ${ }^{5}$ a nominally infinite set of projections onto a UCS structure exists. The theory also indicates that any given projection can be quantified by iteratively convergent algorithms such as the numeric mapping-error minimization adopted in the presented analysis. The UCS reference template for both visual and numeric uniformity is used firstly as a basis for data system linearization, and secondly as a basis for equating unit value over the many distinct dimensions of visual effect. This second requirement to equate scalar value across dimensions is already present in the CMF normalization of the CIE system, and it introduces an important constraint on the unit value mapping process. This is because the mapping functions must not only linearize the model in each dimension of the relationship; they must also generate an equated numeric product in each dimension. The equated product is of course the required unit scalar value constant, and the constraint can be satisfied by holding the causal parameters in balanced inter dimensional equivalence in a process known as neutral axis or "grey scale" tracking.

We can for example use grey scale tracking to further isolate and equate the component response scales as follows: For a given adaptation state, experiments are possible that quantify all points on the visual neutrality axis. The CMF defined proportionate responses are thereby held constant at balanced equivalence, and the opponency responses are held quiescent at their null point. The scalar cone-outputs are thus isolated as the 
THE MATRIX MAPPING HAS TWO DISTINCT STAGES

Stage 1: Quantify the scalar characteristic of each parametric effect.

Stage 2: Quantify and describe all possible cross dependencies.

A THREE DIMENSIONAL EXAMPLE :-

\begin{tabular}{|c|c|c|c|}
\hline & \multicolumn{3}{|c|}{ SCALAR DOMAIN BOUNDARY } \\
\hline $\begin{array}{l}\text { THE SET of ALL POSSIBLE CAUSES by } \\
\text { COMBINING PARAMETERS } A, B, C\end{array}$ & \multicolumn{2}{|c|}{ TWO STAGE MAPPING } & $\begin{array}{l}\text { THE SET of ALL POSSIBLE THREE } \\
\text { DIMENSIONAL EFFECTS }\end{array}$ \\
\hline \multirow{6}{*}{$\begin{array}{l}\text { WHICH HAS:- } \\
\text { 1. A dimensionality and axes quantified } \\
\text { by its three causal parameters. } \\
\text { 2. A scalar basis quantified by the unit } \\
\text { cause values of each parameter. } \\
\text { 3. A set of observable effects produced } \\
\text { by altering each parameter, which can } \\
\text { be used experimentally to establish a } \\
\text { descriptive data set. } \\
\text { 4. A relationship with the dimensions of } \\
\text { observed effect, which may be a } \\
\text { complex nonlinear and cross } \\
\text { dependency }\end{array}$} & DEFINE UNITS & $\begin{array}{c}\text { DEFINE } \\
\text { DEPENDENCIES }\end{array}$ & \multirow{5}{*}{$\begin{array}{l}\text { WHICH UNDER THE MAPPING HAS:- } \\
\text { 1. A dimensionality quantified by its } \\
\text { three causal parameters. } \\
\text { 2. A uniform and linear scaling } \\
\text { quantified by the three dimensional } \\
\text { unit value transformation. } \\
\text { 3. A matrix of observed proportionate } \\
\text { effects which are produced by } \\
\text { altering each causal parameter, and } \\
\text { are unambiguously described by } \\
\text { the derived scalar constants. }\end{array}$} \\
\hline & \multirow{3}{*}{$\begin{array}{c}A^{\prime}=f(A) \\
\underset{B^{\prime}=f(B)}{\longrightarrow} \\
C^{\prime}=f(C)\end{array}$} & $\begin{array}{c}\text { For any given effect } \\
X, Y, Z\end{array}$ & \\
\hline & & & \\
\hline & & $z]^{-}$ & \\
\hline & & $\begin{array}{c}\text { Where } X, Y, Z \\
\text { and } A^{\prime}, B^{\prime}, C^{\prime} \text { now share } \\
\text { a common unit value } \\
\text { definition }\end{array}$ & \\
\hline & $\begin{array}{l}\text { Unit Value } \\
\text { Transformation }\end{array}$ & \multicolumn{2}{|c|}{ The Desired Linear Descriptive Metric } \\
\hline
\end{tabular}

FIG. 4. A matrix mapping system for describing nonlinear cross dependencies. The model first establishes a potentially nonlinear unit value metric that describes the magnitude of observed incremental effect for each causal parameter. It then quantifies the apparent cross dependencies under the resulting linearized and equated unit system as a strictly constant matrix of effects. [Color figure can be viewed in the online issue, which is available at www.interscience.wiley.com.]

exclusively predominant response and they can be quantified by direct experiment. We might for example quantify the response of just perceptible achromatic luminosity difference at increasing levels of stimulus power density. In principle, the three individual cone-output characteristics are then determined by projecting the experimental stimulus values that quantify threshold difference onto their linear and inter dimensionally equivalent UCS definition. The intent is to follow Kuehni's suggestion, ${ }^{11}$ that the phenomena of just noticeable difference at the neutral axis, and supra-threshold chromatic difference should be analyzed as fundamentally distinct phenomena, using appropriately definitive models and data sets.

\section{FINDING THE LINEAR MODEL BY NONLINEAR PROJECTION}

The grey-scale tracking analysis is a special case of the vector space projection method known as POCS (see also Appendix). The additional constraints of neutral axis tracking enable multi-dimensional nonlinear cross dependency relationships to be resolved, and the resolution is provided by a projective mapping of scalar cause onto scalar effect consisting of two distinct components. A set of potentially nonlinear and simultaneously acting single dimension functions is used to transform unit scalar value at the inter-domain boundary that links cause to effect, and an axiomatically distinct matrix of cross dependency ratios then quantifies all possible vector sum additive effects. As illustrated in Fig. 4, the transformation first relinearizes and re-equates unit value at the inter-domain boundary by reference to observed increments in the neu- tral axis visual responses: Having thus established a verifiable linear model, this in turn enables the cross dependency matrix relationship to be established as an independent systematic constant. In combination these two transformations establish a mapping by nonlinear projection onto the set of hypothetical properties represented by the axiomatically convex UCS structure.

This decomposition establishes a clear theoretical basis for calibrating the nonlinear colour reproduction characteristics of computer monitor screens. It also explains the critical importance of grey scale tracking, the essentially empirical success of the ICC profiling approach to colour calibration, ${ }^{13}$ and the accurate and comprehensively tested CRT calibration reported in reference. ${ }^{14}$

\section{PRACTICAL REALIZATION OF THE ANALYTICAL METHOD}

The component visual responses are first classified as either multi-dimensionally vector additive or intra-dimension scalar phenomena, and the specific sets and subsets of component responses are then identified classified and isolated for experimental investigation using a notional hierarchy of inter-domain boundaries as in Figs. 1 and 2. A set of reference UCS structures is then declared, whose members are templates for constructing specific colorimetric domain sub-models. The individual component responses are then calibrated against a specific component of the UCS reference system, which in principle has a dimension of descriptive scalar value for each visually distinct causal dimension. The overall model is initially unpopulated, and it consists of empty (that is to say 
unquantified) proportionate value sub-spaces, and scalar mapping projections. In principle each such empty component can then be populated by mapping experimental dataset members expressed in terms of unit cause onto the relevant spatial template. The additive responses of visual equivalence and multi-dimensional proportionate value colour mixture are then quantified by datasets derived under a constant scaling definition from visual matching experiments; and the visual scaling responses are quantified by visual difference magnitude estimation experiments where ratio value differences are held constant.

The product of each projective scalar mapping is in effect a redefinition of unit value and a default 1:1 mapping relationship in each dimension is assumed, thereby implying that unit redefinition is not required at the relevant inter domain boundary. The presented colorimetric analysis is thus directly equivalent both numerically and visually to the standard CIE $X Y Z$ tristimulus value analysis unless a statistically validated mapping-error minimization reveals that the relationship under investigation can be better described by an alternative mapping. The projections are thus optimized until at some level of statistical confidence they either demonstrably characterize a constant relationship that is common to all of the observed instances of cause and effect; or the iteration establishes that no such constant scalar or proportionate relationship exists as a property of the experimental data set.

Few members of the set of all possible projections will actually represent the properties of the relevant experimental data sets. The intent is therefore to design experiments to either confirm or reject the hypothesis on a case by case basis that under an appropriate projection, all instances of observed effect represented in a given dataset can be characterized by a specific set of validated constants. Tangible benefit from this process will then be demonstrated by accumulating additional statistically verified constants of the visual response. On the other hand, the alternative debating stance will steadily gain weight by eliminating the potentially important and more likely candidate constants, such as the unit values that may be characteristic of hue chroma and lightness difference.

\section{SOME CANDIDATE VISUAL RESPONSE CONSTANTS}

To investigate the possibility that the CMFs may be members of a larger super-set of potentially important visual response constants, we might perhaps study the visual additivity constant. In principle, any confirmed pattern of wavelength or stimulus power dependent super or sub-additive visual responses establishes a metamerically equivalent and spectrally distinct definition of the true CMF constant, and the dataset of primary triplet matches that quantify the CIE Standard Observer establishes just one of many such observer-metameric alternatives. In addition to studying the additivity constant, we might also design experiments based on the Bezold-Brücke (BB) hue shift at constant stimulus wavelength; ${ }^{9,15}$ on the Helmholtz-Kohlrausch (HK) brightness effect in hetero chromatic stimulus matching; ${ }^{8}$ and on sets of complementary colours, whose ratio-value properties are described by Pridmore. ${ }^{16}$

Apparent super- and sub-additive effects and errors in transformability between alternative primary CMF determinations have been reported by Thornton in part III of his article series, ${ }^{10}$ and if such responses are confirmed to be present, this would undermine the key assumption of colorimetry that the property of additivity is invariant over wavelength. Any deviations from constant additivity can be characterized by a projective mapping that demonstrably restores transformability. A mapping of this type that restores transformability between the Thornton PC CMF dataset and the CIE Standard Observer CMF dataset is quantified in the two part reference. ${ }^{17,18}$

In principle, a set of invariant vector directions whose members represent the responses to constant wavelength stimuli could also be established as a characteristic visual response constant by calibrating out the $\mathrm{BB}$ hue shift using a projective mapping. It may also be possible to derive and validate an appropriate experimental definition of unit threshold hue difference as a function of wavelength at constant stimulus power density. The intent is again to calibrate out the nonuniformity of definition (this time for difference of spectral vector direction), thereby adding another quantified unit response mapping onto the visually uniform master space. It is probably also worth investigating the phenomena of achromatic luminosity and chromatic brightness. Under the presented analytical hypothesis, and following Kuehni's suggestion, ${ }^{19}$ the brightness response is classified as sequentially super dependent on the unit luminosity response of the sensor system. The suggestion is therefore that a key inter-domain boundary may exist between the achromatic luminosity response and the chromatic brightness response. The characteristic three dimensional unit value of chromatic brightness difference should therefore be established by scalar matrix mapping as a function of the previously quantified unit of achromatic luminosity difference. If experiment then reveals it to be necessary, the unit values for the visual brightness scale and the associated chromatic difference values can then be modeled as significantly different from the luminosity scale and unit difference values along the neutral axis.

\section{AUTHOR'S NOTE}

This set of notes represents a personal view, and the presented ideas have developed gradually over a number of years. They also in part reflect the views of a group of colleagues $^{20}$ with whom I have held an on-going discussion of my ideas. They certainly haven't always agreed with me, but the interaction has been stimulating and often helpful and I acknowledge and welcome their input.

\section{APPENDIX: AXIOMS THEOREMS PROOFS AND METHODS}

The analysis depends on some important axioms theorems and proofs of affine geometry that are reported in articles 
by Krantz, ${ }^{6,7}$ which are subtitled respectively 'Representation Theorem for Grassmann Structures', and 'Opponent Colors Theory'. These articles equate and axiomatize Grassmann's laws of colour mixture with a convex cone in a true vector space, and they provide worked proofs, and theorems to support the stated definitions. In the quoted articles these spaces are referred to as Grassmann Structures; however in this article I prefer to call them Uniform Colour Systems or UCS structures.

To quote the second article,

A Grassmann Structure is a quadruple $\left\langle A, \oplus,{ }^{*}, \sim\right\rangle$, such that $\left\langle A, \oplus,{ }^{*}\right\rangle$ is a convex cone (Axioms 1 and $2) ; \sim$ is an equivalence relation (Axiom 3); and Grassmann's additivity law holds (Axioms 4 and 5). The set A is interpreted as the set of all possible coloured lights, or spectral radiance distributions; $\oplus$ and * are interpreted respectively as additive colour mixture, and multiplication by positive scalars; and $\sim$ is the binary relationship of metameric matching'.

By both formal declaration and experimental intent the linear and uniform scaling in the reference UCS structures is equated with incremental uniformity in all dimensions of the visual response. The axiomatic convexity of the reference structures then enables the de-convolution method known as 'projection onto convex sets' or POCS. The method of unit value redefinition by nonlinear projection and neutral axis tracking used in these notes appears to be a special case of POCS, and the special case is validated by demonstrating that the product of the simultaneously-acting set of unit value defining functions quantifies a true constant of the data set under analysis. POCS theory further indicates that the iterative refinement of a given projection will always converge if smooth functions are used, and the 'onto' vector space is demonstrably convex. The presented iterative error-minimization method for refining the set of mapping functions will thus only fail to generate a statistically valid product if the potential constant under investigation does not exist as a property of the relevant data set.

Each mapping function from scalar cause onto scalar effect is validated as a single dimension UCS structure or UCS Code when its product demonstrably satisfies the same set of axioms by characterizing a set of strictly uniform and constant visual scales. A nominally infinite set of UCS Code mapping projections onto the reference space then exists, but only a small subset of these (thought to be essentially empty by the sceptics) will quantify a possible constant of the visual response. A projection only characterizes a true constant when all members of a relevant data set are exactly mapped onto the reference UCS structure by a single definitive set of scalar mapping functions.

I emphasize here the dual role of UCS structures and their defining axioms. They exist both as proven context free mathematical constructs and as applied in the current context, where I use both the structures and the axioms indirectly via the UCS declaration and numeric mappingerror minimization as constraining rules for validating both linear and nonlinear mapping relationships.

1. Laming D. Measurement of Sensation (Oxford Psychology Series). Oxford, UK: Oxford University Press; 1997. p 14. et seq. ISBN 0198523424 Publ.

2. Wyszecki G, Stiles WS. Color scaling. In: Wyszecki \& Stiles Color Science, 2nd edition. New York: Wiley Interscience; 2000. p 488.

3. Fairchild MD. Refinement of RLAB Color Space. Color Res Appl 1996;22:174-188.

4. Berns RS. A generic approach to color modeling. Color Res Appl 1997;22:318-325.

5. Stark H, Yang Y. 'Vector Space Projections: A Numerical Approach...' Wiley Series in Telecommunications and Signal processing. Publ. New York: Wiley Interscience, Chapter 2 p 33.

6. Krantz DH. Representation theorem for grassmann structures, part I. J Math Psychol 1975:12:283-303.

7. Krantz DH. Opponent-colors theory. J Math Psychol 1975;12:304327.

8. Alman DH, Breton ME, Barbour J. New results on the brightness matching of heterochromatic stimuli. J Illum Eng Soc 1983;12:268274.

9. Pridmore RW. Bezold-Brucke effect exists in related and unrelated colors and resembles the Abney effect. Color Res Appl 2004;29: 241-246.

10. Thornton WA. Toward a more accurate and extensible colorimetry, Part III. Color Res Appl 1992;17:240-262.

11. Kuehni RG. Threshold color differences compared to supra-threshold color differences. Color Res Appl 2000;25:226-229.

12. Wyszecki G, Stiles WS. The Brown-MacAdam Ellipsoids. In: Wyszecki \& Stiles Color Science, 2nd edition. New York: Wiley Interscience; 2000. p 313.

13. Specification ICC. 1.2004-10 (Profile Version 4.2.0.0). Reston VA 20191 USA: International Color Consortium, 2004.

14. Oulton DP, Boston CJ, Walsby R. IS\&T/SID 4th International Imaging Conference, Scottsdale Arizona, Nov. 1996; pp 14-19.

15. Pridmore RW. 'Bezold-Brucke hue shift as functions of luminance level etc .... Vis Res 1999;39:3873-3891.

16. Pridmore RW. Effects of luminance, wavelength and purity on color attributes. Color Res Appl 2007;32:208-222.

17. Oulton DP. The properties of multiple CMF determinations using alternative primary sets. I. Evidence and modeling. Color Res Appl 2004:29:273-284.

18. Oulton DP. The properties of multiple CMF determinations using alternative primary sets. II. A data unification methodology. Color Res Appl 2004;29:438-450.

19. Kuehni RG. An opponent color model for the Sanders-Wyszecki Helmholtz-Kohlrausch effect data set. Color Res Appl 2000;24:292-293.

20. Personal correspondence with, Danny Rich, Michael Brill, Rolf Kuehni, Steven Westland, David Krantz, David Foster and Alan Robertson. 